\title{
THE DEVELOPMENT OF AN INHOMOGENEOUS DAMAGE FIELD IN AN ELASTIC-PLASTIC MODEL
}

\author{
H. L. $\mathrm{LI}^{1}$, Y. L. BAI ${ }^{1}$ and M. F. XIA ${ }^{1,2}$ \\ ${ }^{1}$ Laboratory for Nonlinear Mechanics of Continuous Media, Institute of Mechanics, Chinese Academy of Sciences, \\ Beijing 100080, China \\ ${ }^{2}$ Department of Physics, Peking University, Beijing 100871, China
}

\begin{abstract}
By making use of the evolution equation of the damage field as derived from the statistical mesoscopic damage theory, we have preliminarily examined the inhomogeneous damage field in an elastic-plastic model under constant-velocity tension. Three types of deformation and damage field evolution are presented. The influence of the plastic matrix is examined. It seems that matrix plasticity may defer the failure due to damage evolution. A criterion for damage localization is consistent with the numerical results.
\end{abstract}

Keywords—Damage field; Damage localization; Statistical mesoscopic damage; Plasticity.

\section{NOMENCLATURE}

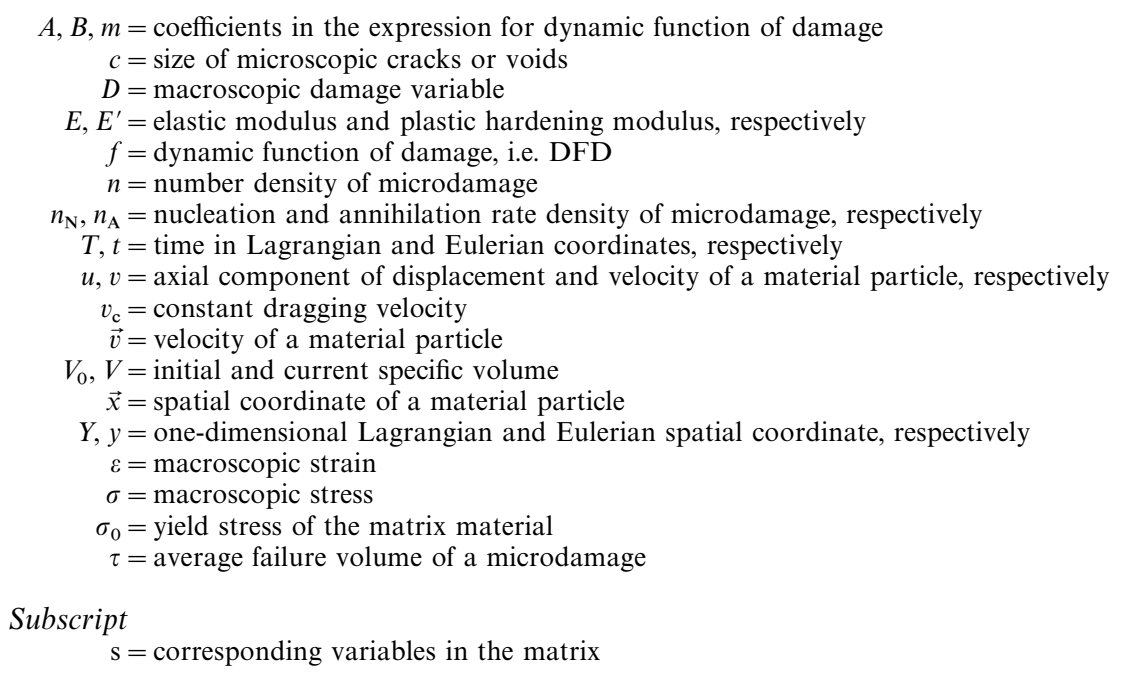

\section{INTRODUCTION}

Many attempts have been made to reveal the mechanisms of material degradation and damage cumulation in order to predict the occurrence of material failure beforehand and avoid disaster. In fracture mechanics, people tried to deal with this problem by investigating one or several macroscopic cracks; while in continuum damage mechanics, researchers tried to treat damage processes based on assumed damage evolution law $[1,2]$. However, great progress achieved recently in experimental techniques make it possible to observe the real process of nucleation, growth and coalescence of numerous microscopic cracks or voids, count the number and measure the sizes by metallographical means. This provides a clear physical background of the microscopic damage evolution process. The enormous microcracks or voids observed in materials suggest collective 
effects of microdamage [3-5]. Thus, an approach of statistical mesoscopic damage mechanics is proposed [3-6]. In this way, we can take advantage of microscopic experimental observations and treat damage evolution more realistically.

Based on the experimental data from microscopic observations, Curran et al. [3] proposed a nucleation and growth (NAG) model to statistically describe the microdamage evolution. Bai and co-workers examined the statistical evolution of microcracks in an aluminium alloy containing second-phase particles subjected to impulse loading $[4,5]$. The kinetics by which nucleation and growth of microcracks proceed are investigated based on the experimental data and a statistical formulation [6,7]. Recently, efforts have been made to combine these results of statistical mesoscopic damage mechanics with the equations of continuum mechanics to give some theoretical prediction of the failure process, e.g. damage localization, on a macroscopic level [8]. This is the motivation for the present study.

On the other hand, especially for most metals, the plastic deformation of the matrix material usually occurs simultaneously with the microdamage evolution. What are their coupling effects on the failure process of materials? This is one of the key points of our present investigation.

Below, we briefly introduce the damage evolution equation derived from the theory of statistical mesoscopic damage mechanics [8]. Then, the application of these equations to the case of constantvelocity tension is especially emphasized. In this case, we consider not only the evolution of microdamage, but also the plastic deformation of the matrix material. A criterion for damage localization suggested in Ref. [8] is examined. We found that this is consistent with the results of our numerical examples.

\section{EVOLUTION EQUATION OF DAMAGE FIELD}

We investigate a material particle which is large enough to contain a number of microdamages with different sizes, but is small enough to be thought of as a point on a macroscopic level.

First, on a mesoscopic level, numerous cracks and voids within a material particle are considered. The number density $n(t, c, \vec{x})$ is used to describe the collective behaviour of all these microdamages, i.e. the number of cracks or voids with sizes between $[c, c+\mathrm{d} c]$ at time $t$ is given by $n(t, c, \vec{x}) \mathrm{d} c$, where $\vec{x}$ is the spatial coordinates of the material particle. From the viewpoint of statistical mesoscopic damage mechanics $[5,6]$, the evolution equation of number density can be derived based on the balance law of the amount of microdamage in the phase space $(c, \vec{x})$

$$
\frac{\partial n}{\partial t}+\frac{\partial(n \dot{c})}{\partial c}+\nabla \cdot(n \vec{v})=n_{\mathrm{N}}-n_{\mathrm{A}}
$$

where $\dot{c}$ is the growth rate of microdamage, $\vec{v}$ is the velocity of the material particle, $\nabla$ is the gradient operator. $n_{\mathrm{N}}$ and $n_{\mathrm{A}}$ are nucleation and annihilation rate densities of microdamage, respectively.

Second, on a macroscopic level, a continuum damage field is under consideration. The damage variable of a material particle is defined as:

$$
D=\int_{0}^{\infty} n(t, c, \vec{x}) \tau \mathrm{d} c
$$

where $\tau$ is the average failure volume of a microdamage with current size $c$, e.g. $\tau=(\pi / 6) c^{3}$ for spherical voids. After multiplying Eq. (1) by $\tau$ and integrating it, the evolution equation of the 
damage field can be established to be:

$$
\frac{\partial D}{\partial t}+\nabla \cdot(D \vec{v})=f
$$

where

$$
f=\int_{0}^{\infty} n_{\mathrm{N}} \tau \mathrm{d} c+\int_{0}^{\infty}\left(n A \tau^{\prime}-n_{\mathrm{A}} \tau\right) \mathrm{d} c \quad \text { and } \quad \tau^{\prime}=\frac{\partial \tau}{\partial c}
$$

$f$ is referred to as the dynamic function of damage (DFD). This is a function concerning all possible mesoscopic processes, e.g. nucleation, growth and coalescence of microdamage. Especially, $f$ can be expressed by the sum of a nucleation term and other terms (e.g. growth and coalescence of microdamage).

In the case of a one-dimensional state (along the $y$-axis), Eq. (3) can be reduced to

$$
\frac{\partial D}{\partial t}+v \frac{\partial D}{\partial y}+D \frac{\partial v}{\partial y}=f
$$

where $v$ denotes the axial component of the particle velocity. Noting that the above derivation is in Eulerian coordinates $(t, y)$, we may transform the formulation into Lagrangian (material) point space in terms of the time and spatial coordinates $(T, Y)$, i.e.

$$
\frac{\partial D}{\partial T}+D \frac{V_{0}}{V} \frac{\partial v}{\partial Y}=f
$$

where $T=t, Y=y-\int_{0}^{T} v \mathrm{~d} T, V_{0} / V$ is the ratio of an initial specific volume and its current volume. (For a more detailed discussion, see Ref. [8].)

\section{EVOLUTION OF INHOMOGENEOUS DAMAGE FIELD}

\section{Basic equations}

In the present study, attention is focused on the evolution of an inhomogeneous damage field in an elastic-plastic material subjected to constant-velocity tension. To simplify the analysis, only a one-dimensional state is considered. In this case, the damage field equation can be combined with the continuum and momentum equations to form the following system of equations:

$$
\begin{gathered}
\frac{\partial D}{\partial T}+D \frac{V_{0}}{V} \frac{\partial v}{\partial Y}=f \\
\frac{\partial V}{\partial T}-V_{0} \frac{\partial v}{\partial Y}=0 \\
\frac{\partial v}{\partial T}-V_{0} \frac{\partial \sigma}{\partial Y}=0
\end{gathered}
$$

where $\sigma$ is the axial tension stress.

The nominal stress $\sigma$ on a damaged material particle is related to the real stress $\sigma_{\mathrm{s}}$ in the matrix by the following conventional relation:

$$
\sigma=\sigma_{\mathrm{s}}(1-D)
$$

Concerning the constitutive relationship of the matrix material, we assume an elastic-plastic 
hardening relation

$$
\sigma_{\mathrm{s}}= \begin{cases}E \varepsilon_{\mathrm{s}} & \left(\varepsilon_{\mathrm{s}} \leqslant \varepsilon_{0}\right) \\ \sigma_{0}+E^{\prime}\left(\varepsilon_{\mathrm{s}}-\varepsilon_{0}\right) & \left(\varepsilon_{\mathrm{s}}>\varepsilon_{0}, \dot{\varepsilon}>0\right)\end{cases}
$$

where $\sigma_{0}$ is the yield stress of the matrix, $\varepsilon_{0}=\sigma_{0} / E$, and $E, E^{\prime}$ are the elastic modulus and hardening modulus of plastic deformation, respectively (where $E=\bar{E}, E^{\prime}=\bar{E}^{\prime}, \sigma=\bar{\sigma}_{0}, \varepsilon_{0}=\bar{\varepsilon}_{0}$ for one-dimensional stress state;

$$
E=\frac{1-v}{(1+v)(1-2 v)} \bar{E}, \quad E^{\prime}=\frac{\bar{E}}{3}\left(\frac{1}{1-2 v}+\frac{2}{1+v} \frac{\bar{E}^{\prime}}{\bar{E}}\right), \quad \sigma_{0}=\frac{1-v}{1-2 v} \bar{\sigma}_{0}, \quad \varepsilon_{0}=(1+v) \bar{\varepsilon}_{0}
$$

for one-dimensional strain state approximately, and $v$ denotes Poisson's ratio). If unloading occurs, it follows a path parallel to the elastic one from the unloading point.

Under the constant-velocity tension, the boundary conditions at two ends of a specimen are expressed as follows

$$
\begin{array}{ll}
u=0 & (\text { at } Y=0) \\
u=v_{\mathrm{c}} T & (\text { at } Y=L)
\end{array}
$$

where $u$ is the displacement component, $v_{\mathrm{c}}$ is the constant dragging velocity, and $L$ is the distance between the two ends of the specimen. Also, an inhomogeneous distribution of damage $D_{0}=$ $D(0, Y)$ and free stress $\sigma(0, Y)$ are presumed to be the initial conditions.

\section{Analysis}

We consider the one-dimensional strain state. For the case of small quasi-static deformation, the problem can be further simplified and investigated analytically. In view of the fact that $\partial \sigma / \partial Y=0, \varepsilon=\partial u / \partial Y)=\left(V / V_{0}\right)-1$, the system of Eqs (6)-(8) can be reduced to the following form:

$$
\frac{\partial D}{\partial T}+\frac{D}{1+\varepsilon} \frac{\partial \varepsilon}{\partial T}=f, \quad \sigma=\sigma(T)
$$

A geometric relation derived from the definition of $D$ gives

$$
\varepsilon=\left[\left(D-D_{0}\right)+\varepsilon_{\mathrm{s}}\left(1-D_{0}\right)\right] /(1-D)
$$

with the initial damage denoted by $D_{0}$.

The dynamic function of damage (DFD) $f$ depends on the mesoscopic mechanisms of microdamage evolution. Usually, it can be expressed in a closed function of macroscopic stress and damage, e.g. Ref. [9]. The results of statistical mesoscopic damage mechanics and experimental investigation on an aluminium alloy [4,5] indicate that the DFD $f$ may include two parts. The first is the contribution of random nucleation, which seems to be dependent on macroscopic stress only [4]; the second is due to the growth and coalescence process of microdamage, which both depend on the macroscopic stress as well as the damage variable $D$ itself, and refers to compound damage $[7,10]$. Thus, we take the following form of DFD as a sample function

$$
f=\left(A^{*}+B^{*} \cdot D^{m}\right) \cdot \sigma / E
$$

where $A^{*}, B^{*}$ and $m$ are fitting parameters of experimental data.

Combining Eqs (11)-(13), we obtain the governing equations as follows:

$$
\left\{\begin{array}{l}
(1-D)^{2} \dot{\varepsilon}+g_{1}\left(D, \varepsilon, \varepsilon^{*}\right) \dot{D}=g_{2}(\dot{\sigma}, D, \varepsilon) \\
D \dot{\varepsilon}+(1+\varepsilon) \dot{D}=(1+\varepsilon) f
\end{array}\right.
$$


where $(\cdot)=\partial / \partial T$,

$$
g_{1}\left(D, \varepsilon, \varepsilon^{*}\right)
$$

$$
= \begin{cases}2 D-D-1-2(1-D) \varepsilon & \text { (elastic stage) } \\ 2 D-D_{0}-1-2(1-D) \varepsilon+\left(1-D_{0}\right)\left(\varepsilon_{0}-\frac{\sigma_{0}}{E^{\prime}}\right) & \text { (plastic hardening stage) } \\ 2 D-D_{0}-1-2(1-D) \varepsilon+\left(1-D_{0}\right)\left[\varepsilon^{*}-\frac{\sigma_{0}}{E}-\frac{E^{\prime}}{E}\left(\varepsilon^{*}-\varepsilon_{0}\right)\right] & \text { (plastic unloading stage) }\end{cases}
$$$$
g_{2}(\dot{\sigma}, D, \varepsilon)= \begin{cases}\frac{\dot{\sigma}}{E}\left(1-D_{0}\right) & \text { (elastic and plastic unloading stage) } \\ \frac{\dot{\sigma}}{E^{\prime}}\left(1-D_{0}\right) & (\text { plastic hardening stage })\end{cases}
$$

where $\varepsilon^{*}$ is the maximum strain in the hardening phase. Thus,

$$
\left\{\begin{array}{l}
\dot{\varepsilon}=(1+\varepsilon) \cdot\left[g_{2}(\dot{\sigma}, D, \varepsilon)-f \cdot g_{1}\left(D, \varepsilon, \varepsilon^{*}\right)\right] /\left[(1+\varepsilon)(1-D)^{2}-D g_{1}\left(D, \varepsilon, \varepsilon^{*}\right)\right] \\
\dot{D}=\left[(1-D)^{2}(1+\varepsilon) f-D \cdot g_{2}(\dot{\sigma}, D, \varepsilon)\right] /\left[(1+\varepsilon)(1-D)^{2}-D g_{1}\left(D, \varepsilon, \varepsilon^{*}\right)\right]
\end{array}\right.
$$

with the initial condition given by $D_{0}(0, Y)$ and the boundary condition given by

$$
\int_{0}^{L} \dot{\varepsilon}(T, Y) \mathrm{d} Y=v_{\mathrm{c}}
$$

Condition (16) expresses the spatial coupling effect of the deformation field.

The inhomogeneity of the damage field can be defined by $(\partial D / \partial y) / D$. Thus, the condition

$$
\frac{\partial}{\partial t}\left[\left(\frac{\partial D}{\partial y}\right) / D\right] \geqslant 0
$$

indicates a transition in the rate of damage inhomogeneity to positive. This may trigger the occurrence of damage localization. In fact, it can be derived from Eq. (17) that

$$
\left(\frac{\partial\left(\frac{\partial D}{\partial y}\right)}{\partial t}\right) /\left(\frac{\partial D}{\partial y}\right) \geqslant\left(\frac{\partial D}{\partial t}\right) / D
$$

which shows that the relative increase in damage gradient becomes greater than the relative increase in damage itself. As a result, a criterion for damage localization can be derived from Eq. (17) with the help of the assumption of small quasistatic deformation [8],

$$
f_{D}-\dot{\varepsilon} \geqslant f / D
$$

and a lower bound

$$
f_{D} \geqslant f / D
$$

where $f_{D}$ denotes $\partial f / \partial D$. 


\section{NUMERICAL RESULTS AND DISCUSSION}

In Figs $1-3$, for the same initial damage distribution

$$
D_{0}(Y)=0.01 \exp \left[-100 \cdot\left(\frac{Y}{L}-\frac{1}{2}\right)^{2}\right]
$$

three samples with the following parameters are shown: (i) $A=\left(L / v_{\mathrm{c}}\right) A^{*}=1, B=\left(L / v_{\mathrm{c}}\right) B^{*}=0$, $\sigma_{0} / E=0.1$, and $E^{\prime} / E=0.2$, this corresponds to the case where only the influence of microdamage nucleation is accounted for; (ii) $A=1, B=500, \sigma_{0} / E=0.1, E^{\prime} / E=0.2$, this is the case where contributions of nucleation, growth and coalescence are all included; (iii) $A=1, B=500, \sigma_{0} / E=$ $0.1, E^{\prime} / E=0.05$, in this case the constitutive curve of the matrix can be approximately viewed as an elastic-perfectly plastic one. It can be inferred that three kinds of features manifest themselves,
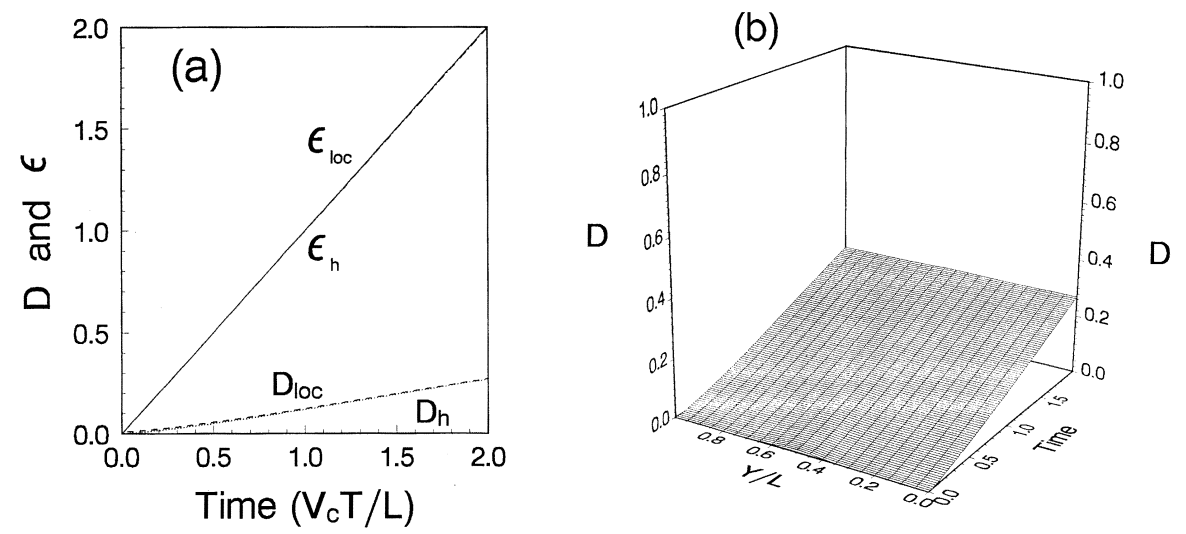

Fig. 1. Homogeneous damage evolution $\left[A=1, B=0, m=2, \sigma_{0} / E=0.1, E^{\prime} / E=0.2\right.$, initial damage distribution given by Eq. (20)]. (a) $D_{\mathrm{h}}, \varepsilon_{\mathrm{h}}=$ average values of $D, \varepsilon ; D_{\mathrm{loc}}, \varepsilon_{\mathrm{loc}}=D$ and $\varepsilon$ at $Y=L / 2$; (b) evolution process of the damage field.
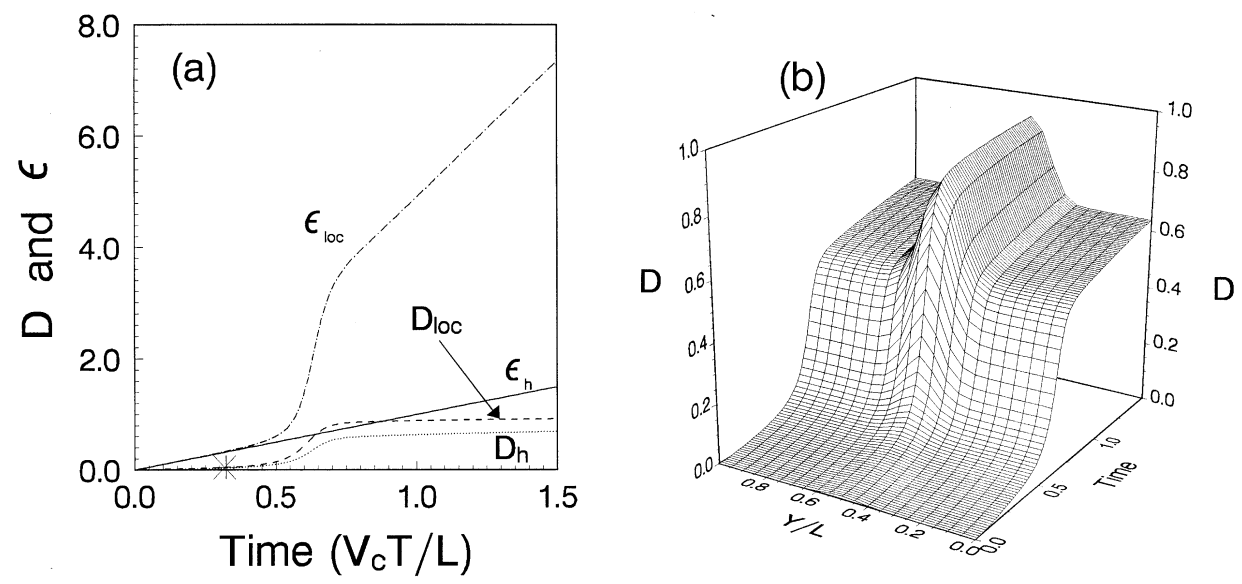

Fig. 2. Deformation and damage localization $\left[A=1, B=500, m=2, \sigma_{0} / E=0.1, E^{\prime} / E=0.2\right.$, initial damage distribution given by Eq. (20)]. (a) $D_{\mathrm{h}}, \varepsilon_{\mathrm{h}}=$ average value of $D, \varepsilon ; D_{\mathrm{loc}}, \varepsilon_{\mathrm{loc}}=D$ and $\varepsilon$ at $Y=L / 2 ;$ (b) evolution process of the damage field [,$+ \times$ are the times predicted by criteria (18) and (19), respectively]. 

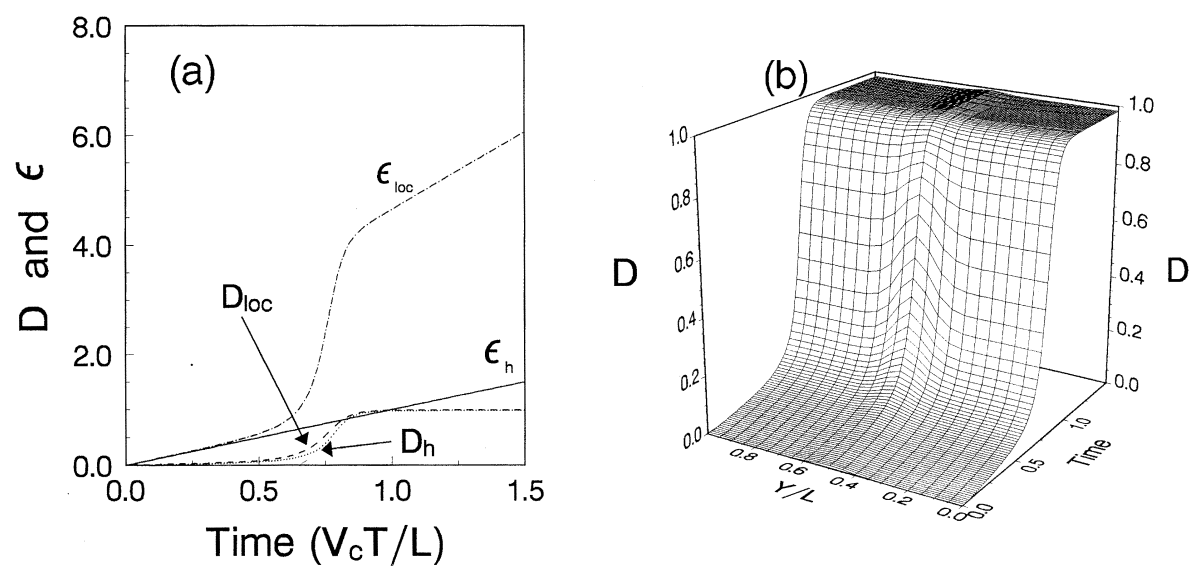

Fig. 3. Deformation localization $\left[A=1, B=500, m=2, \sigma_{0} / E=0.1, E^{\prime} / E=0.05\right.$, initial damage distribution given by Eq. (20)]. (a) $D_{\mathrm{h}}, \varepsilon_{\mathrm{h}}=$ average value of $D, \varepsilon ; D_{\mathrm{loc}}, \varepsilon_{\mathrm{loc}}=D$ and $\varepsilon$ at $Y=L / 2$; (b) evolution process of the damage field.

respectively: For case (i), although the initial distribution of the damage field is inhomogeneous, this kind of inhomogeneity will not become more severe as time elapses, and the deformation and damage field look more likely to remain homogeneous during the whole process, e.g. see Fig. 1. However, for case (ii), the trend for the initial damage to become inhomogeneous is curbed by the whole homogeneous deformation at the beginning. But after a period of time, the damage within the domain with more severe initial damage increases much more rapidly than outside. This tends to dominate the final failure process and cause the macroscopic stress to drop sharply (see Fig. 2). Case (ii) occurs for large values of $B$ and relatively large values of $E^{\prime} / E$, and appears to be a more dangerous case owing to damage localization. Furthermore, for case (iii), where $E^{\prime} / E$ is very small, only deformation localization occurs, whereas the damage field becomes quite homogeneous even at the final stage of deformation (see Fig. 3).

We further examine the influences of matrix plasticity by comparing the results for examples with different values of $\sigma_{0} / E$ and $E^{\prime} / E$ shown in Figs 2-4. It seems that the effect of yield stress $\sigma_{0} / E$ on the damage localization is not monotonous, especially for the cases where the plastic hardening modulus is low. In fact, if $\sigma_{0} / E$ is very high or very low, damage localization may occur; however, once $\sigma_{0} / E$ is in-between somewhere, only deformation localization may take place. On the other hand, for the same value of $\sigma_{0} / E$, when $E^{\prime} / E$ becomes rather large, damage localization will manifest itself for a slightly inhomogeneous initial damage field.

The macroscopic stress curves versus time are given in Fig. 5. When the matrix plasticity is taken into account, the curves look more like the stress-strain curves observed in experiments of one-dimensional tension. In particular, even though the matrix is elastic-plastic hardening, the macroscopic stress $\sigma$ still drops when the damage field is fully developed. This shows the significant influence of the damage evolution process.

It is also found that the matrix plasticity delays the failure due to damage evolution, when compared with the pure elastic case (Fig. 5). Actually, the inhomogeneity of deformation and damage field for plastic cases is not so strong as in the pure elastic case.

The corresponding locations given by the criteria (18) and (19) are shown in Figs 2 and 4, the obtained results verify the criterion. It is also seen that the occurrence of damage localization in the pure elastic case is earlier than that in the plastic case. 

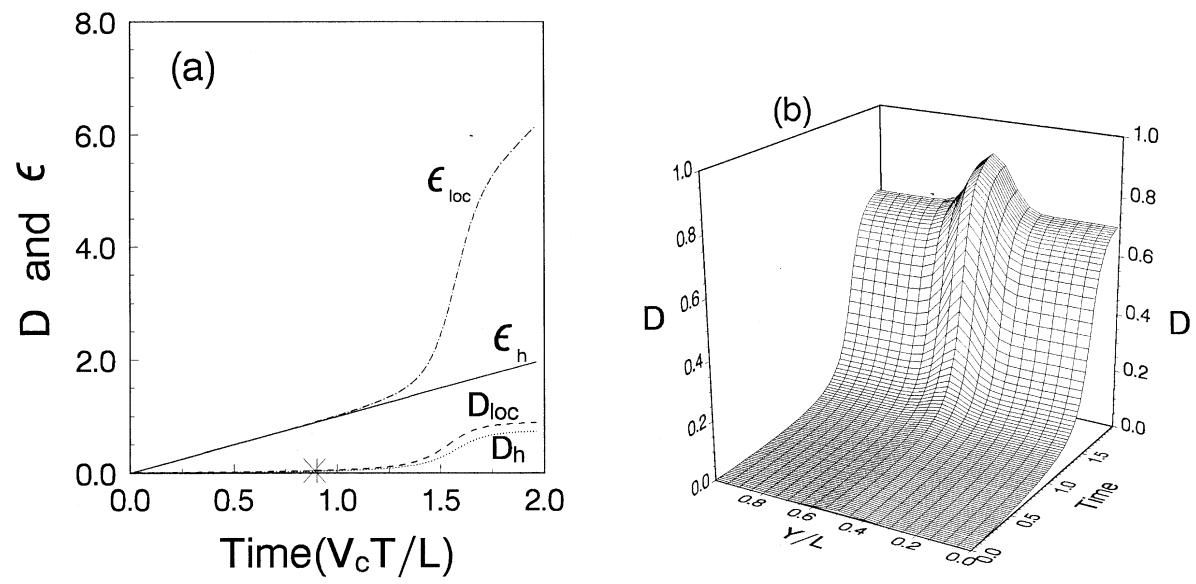

Fig. 4. Deformation and damage localization for a lower yield stress material $[A=1, B=500, m=2$, $\sigma_{0} / E=0.02, E^{\prime} / E=0.05$, initial damage distribution given by Eq. (20)]. (a) $D_{\mathrm{h}}, \varepsilon_{\mathrm{h}}=$ average value of $D$, $\varepsilon ; D_{\mathrm{loc}}, \varepsilon_{\mathrm{loc}}=D$ and $\varepsilon$ at $Y=L / 2$; (b) evolution process of the damage field $[+, \times$ are the times predicted by criteria (18) and (19), respectively].
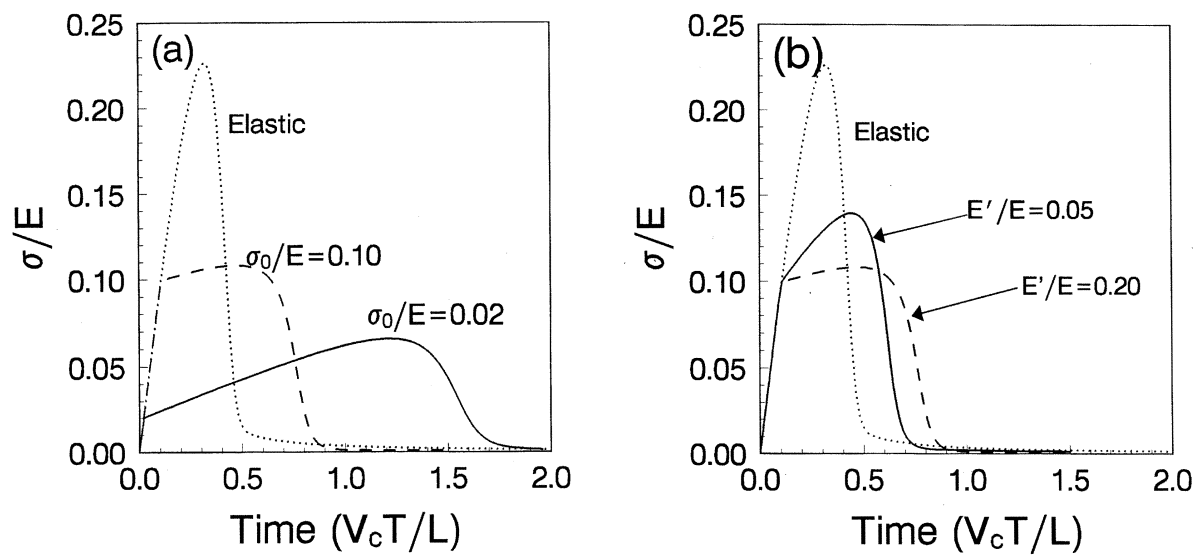

Fig. 5. The change in macroscopic stress versus time $[A=1, B=500, m=2$, initial damage distribution given by Eq. (20)]. (a) $E^{\prime} / E=0.05$; (b) $\sigma_{0} / E=0.1$.

\section{SUMMARY}

The development of an inhomogeneous damage field in an elastic-plastic model under constantvelocity tension is preliminarily examined. Three types of processes connected with the occurrence of deformation and damage localization are discussed. Emphasis is placed on the effects of matrix plasticity. From the numerical results, the effects of the two plasticity parameters on damage localization appear to be different. Under the same plastic hardening modulus, there may be a range of yield stresses, where roughly homogeneous damage develops to failure. But at two extremes of yield stress, damage localization may occur, whilst for the cases with the same yield stress, a higher plastic hardening modulus seems to be more prone to present damage localization. In general, matrix plasticity delays failure due to damage evolution. A criterion for damage 
localization is applied to the cases we considered, which is consistent with the results of numerical examples.

Acknowledgement-The support given by the National Natural Science Foundation of China (19572072 and 19891180) is gratefully acknowledged.

\section{REFERENCES}

1. L. M. Kachanov (1986) Introduction to Continuum Damage Mechanics, Martinus Nijhoff, The Netherlands.

2. J. Lemaitre and A. Plumtree (1979) Application of damage concepts to predict-fatigue failures. $A S M E$ Trans. J. Engng Mater. Tech. 101, 284-292.

3. D. R. Curran, L. Seaman and D. A. Shockey (1987) Dynamic failure of solids. Physics Reports, 147, 253-388.

4. Y. L. Bai, Z. Lin, L. M. Luo and F. J. Ke (1992) Initial development of microdamage under impact loading. ASME Trans. J. Appl. Mech. 59, 622-627.

5. W. S. Han, M. F. Xia, L. T. Shen and Y. L. Bai (1997) Statistical formulation and experimental determination of growth rate of micrometre cracks under impact loading. Int. J. Solids Structures 34, 2905-2925.

6. Y. L. Bai, F. J. Ke and M. F. Xia (1991) Formulation of statistical evolution of microcracks in solids. Acta Mech. Sin. 7, 59-66.

7. Y. L. Bai, W. S. Han and J. Bai (1997) A statistical evolution equation of microcracks and its application. In: Symp. Application of Continuum Damage Mechanics to Fatigue and Fracture (Edited by D. L. McDowell), ASTM STP. 1315, pp. 150-162.

8. Y. L. Bai, M. F. Xia, F. J. Ke and H. L. Li (1998) Damage field equation and criterion for damage localization. Proc. IUTAM Symp. Rheology of Bodies with Defects, Kluwer Academic.

9. Y. L. Bai, M. F. Xia, F. J. Ke and J. Bai (1998) Dynamic function of damage and its implication, fracture and strength of solids. Key Engng Mater. 145-149, 411-420.

10. L. Davison and A. L. Stevens (1972) Continuum measures of spall damage. J. Appl. Phys. 43, 988-994. 